\title{
DEVELOPMENT AND TEST OF NB3SN COS-THETA COILS MADE OF HIGH-JC RRP STRANDS
}

\author{
R. Bossert ${ }^{1}$, G. Ambrosio ${ }^{1}$, N. Andreev ${ }^{1}$, E. Barzi ${ }^{1}$, R. Carcagno ${ }^{1}$, S. Feher ${ }^{1}$, \\ V.S. Kashikhin ${ }^{1}$, V.V. Kashikhin ${ }^{1}$, M.J. Lamm ${ }^{1}$, I. Novitski ${ }^{1}$, \\ Yu. Pischalnikov ${ }^{1}$, C. Sylvester ${ }^{1}$, M. Tartaglia ${ }^{1}$, D. Turrioni ${ }^{1}$, R. Yamada ${ }^{1}$, \\ A.V. Zlobin ${ }^{1}$ \\ ${ }^{1}$ Fermi National Accelerator Laboratory \\ Batavia, Illinois, 60506, USA
}

\begin{abstract}
A series of 1-m long $\mathrm{Nb}_{3} \mathrm{Sn}$ dipole magnets have been built at Fermilab in an attempt to refine the wind-and-react technology for $\mathrm{Nb}_{3} \mathrm{Sn}$ conductor. Models have been made with MJR and PIT strand with varying degrees of success. Subsequently two new dipole "mirror" magnets based on RRP $\mathrm{Nb}_{3} \mathrm{Sn}$ coils were constructed and tested. This paper describes the design, fabrication and test results of those magnets.
\end{abstract}

KEYWORDS: preload, witness samples, mirror, reaction, impregnation.

PACS: 84.71.Ba

\section{INTRODUCTION}

Fermilab is involved in the development of new generation high-field accelerator magnets exploring different design and technological options. One of the possible magnet designs is based on $\mathrm{Nb}_{3} \mathrm{Sn}$ shell-type coils and the wind-and-react technology. A dipole magnet based on this approach was developed at Fermilab ${ }^{1}$, and several 1-m long models were fabricated and tested during 2001-2004. The early dipole models based on Modified Jelly Roll (MJR) strand demonstrated large degradation of magnet quench current ${ }^{2}$. Detailed analysis of possible causes allowed correlating the observed magnet quench performance with large magnetic instabilities in high-Jc MJR Nb${ }_{3}$ Sn strands with large $(\sim 110 \mu \mathrm{m})$ effective filament size used in these models ${ }^{3}$. In order to improve conductor stability, more stable $\mathrm{Nb}_{3} \mathrm{Sn}$ strands with modest Jc and effective filament size of $\sim 50 \mu \mathrm{m}$ produced using powder-in-tube (PIT) technology were used. It allowed the design field of $10 \mathrm{~T}$ to be reached for this magnet ${ }^{4}$. To advance the field to its limit the magnet coil was modified for $0.7 \mathrm{~mm}$ strand without changing the coil layer width or magnet mechanical structure. High-Jc strand with smaller $(\sim 80 \mu m)$ sub-element size produced by Restack Rod Process (RRP) was used in order to mitigate the instability problems and increase the field. Three new half-coils based on this design were 
fabricated and two were tested in a magnetic mirror configuration ${ }^{5}$. This paper describes the parameters of $\mathrm{Nb}_{3} \mathrm{Sn} \mathrm{RRP}$ strand and cable, the details of coil design, magnetic mirror fabrication procedure, and reports the test results.

\section{MAGNET DESIGN}

\section{Coil Cross Section}

Cross section is shown in Figure 1. Coil bore diameter is $43.5 \mathrm{~mm}$. Coil has 2 layers, with 16 turns and two spacers per quadrant in the inner layer and 21 turns and one spacer per quadrant in the outer layer, and separate pole blocks in each layer. Coil layers are separated with $0.4 \mathrm{~mm}$ thick inter-layer insulation. Coil ends have current blocks consistent with the coil straight section and separated by metallic spacers.

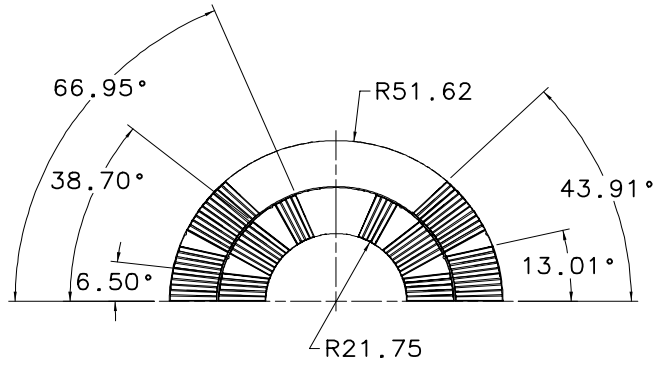

FIGURE 1. Coil cross section.

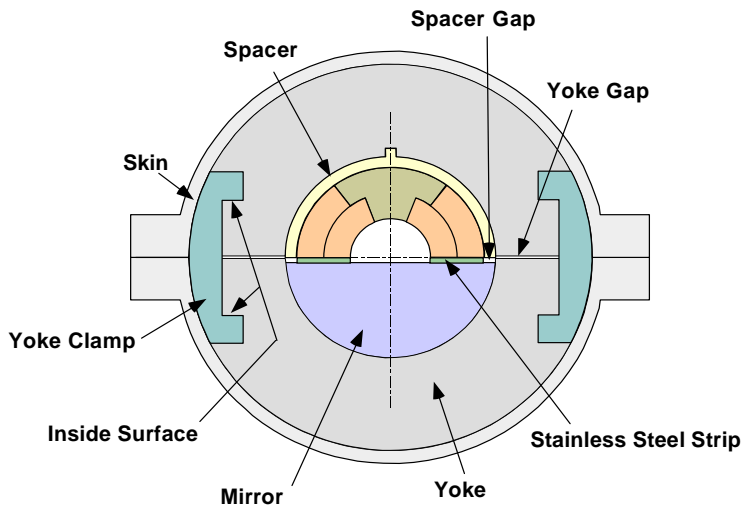

FIGURE 2. HFDM04/HFDM05 Mechanical Structure.

\section{Strand and Cable}

Strand and cable parameters are shown in Table 1. Rutherford type cable with for HFDM04 was manufactured at Lawrence Berkeley Laboratory in one step. The cable for HFDM05 was fabricated in two steps: rectangular cable was made at LBNL and then the final keystone cable after annealing was done at Fermilab. Strand was produced by Oxford Superconductor Technologies (OST) using the RRP process.

TABLE 1. HFDM04 and HFDM05 Strand and Cable Parameters.

\begin{tabular}{|l|c|c|}
\hline Parameter & Unit & Design Value \\
\hline Strand Diameter & $\mathrm{mm}$ & 0.70 \\
\hline Number of sub-elements & & $54 / 61$ \\
\hline Sub-element size & $\mu \mathrm{m}$ & 80 \\
\hline Cu fraction & $\%$ & 50 \\
\hline Cable Midthickness & $\mathrm{mm}$ & $1.20^{*} / 1.24^{* *}$ \\
\hline Cable Width & $\mathrm{mm}$ & $14.36^{*} / 14.24^{* *}$ \\
\hline Cable Keystone angle & $\mathrm{deg}$ & $0.98^{*} / 0.91^{* *}$ \\
\hline Cable Pitch Length & $\mathrm{mm}$ & 111 \\
\hline Number of Strands & & 39 \\
\hline \multicolumn{2}{|l}{ *HFDM04; **HFDM05 } \\
\end{tabular}


The design cable cross-section had a keystone angle of 1.30 degrees, with mid-thickness of $1.240 \mathrm{~mm}$. This specification was subsequently changed, and the actual cables had slightly different keystone angle and mid-thickness (see Table 1). The cable insulation was adjusted to help compensate for these differences. The insulation consisted of $0.125 \mathrm{~mm}$ thick and 12.5 mm wide ceramic tape, spiral wound over the cable with approximately $30 \%$ overlap.

\section{Mechanical Structure}

Mirror mechanical structure is shown pictorially in Figure 2. The iron yoke is split horizontally. Aluminum-bronze spacers surround the coil inside the yoke, ending azimuthally at the coil parting plane. One of the two half-coils in the magnetic mirror configuration is replaced by a solid iron half-cylinder blocks. Stainless steel strips are placed between the mirror and coil parting plane to control coil prestress. Preload is applied by a combination of the aluminum yoke clamps and thick bolted stainless steel skin. There is a gap between yoke halves, which remains open at all stages of construction and testing.

\section{MAGNET FABRICATION}

\section{Coil Winding and Curing}

Each half-coil is wound from a single cable piece without an inter-layer splice. After winding of the inner layer, it is impregnated with liquid ceramic binder and cured. The outer layer is wound on top of the cured inner layer covered with the preformed ceramic interlayer insulation made of 3 layers of $0.125 \mathrm{~mm}$ ceramic sheets. The outer layer is also impregnated with binder and the coil is cured to set the size for reaction. Curing is done at 150C for $1 / 2$ hour in a closed cavity mold made to the nominal coil size. An azimuthal pressure of $35 \mathrm{MPa}$ is applied to the coil to close the mold.

Coil end parts are water jet cut from phosphor bronze tubes. In order to fit the parts onto the uncompressed coil during winding, they were reworked by hand from the original design. This resulted in small spaces between coil end turns and end parts after curing, which were filled with a mixture of ground ceramic tape and CTD-1008 binder.

The midplane current block of the inner coil in HFDM04 had one turn less than nominal. To compensate for that it was wound with a double layer of $0.200 \mathrm{~mm}$ ceramic insulation placed between each turn. The HFDM05 coil has the nominal number of turns.

\section{Reaction}

Reaction is done in a closed fixture set to the nominal coil size. Coil size is set during curing so that the maximum azimuthal pressure during reaction is less than $5 \mathrm{MPa}$ to avoid Sn leaks when the coil expands at reaction temperatures. The target reaction cycle consisted of three ramps, followed by three temperature plateaus. First a ramp at 25C/hrs to 210C, then $100 \mathrm{hrs}$ at $210 \mathrm{C}$, then a ramp at 50C/hrs to 400C, then 400C for $48 \mathrm{hrs}$ and finally a ramp at $75 \mathrm{C} / \mathrm{hrs}$ to $650 \mathrm{C}$, then $650 \mathrm{C}$ for $50 \mathrm{hrs}$. Cooldown from 650C to 20C was programmed to take $48 \mathrm{hrs}$. The oven settings were adjusted to minimize the vertical and back-to-front thermal gradients, and lag time for the retort to reach the temperature of the programmed cycle. Figure 3 shows the actual cycle as read by thermocouples mounted within the oven. 


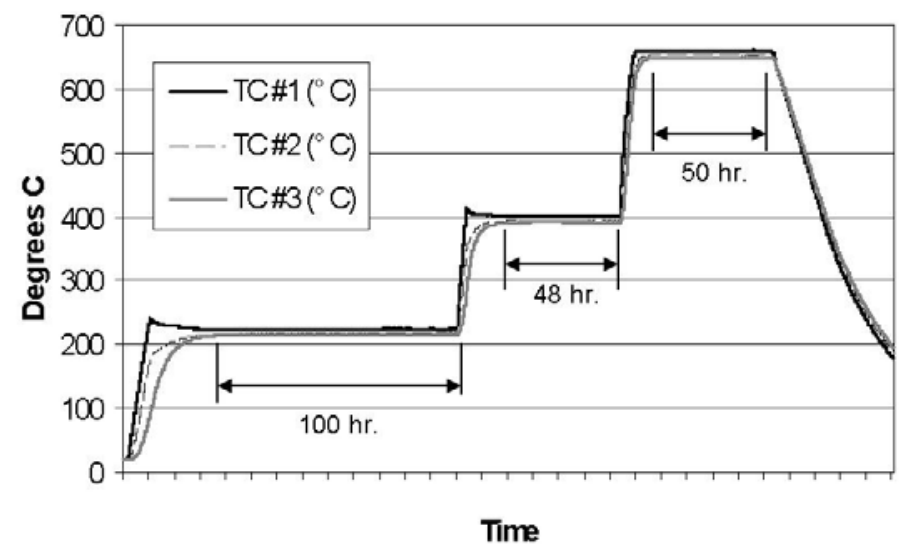

FIGURE 3. Reaction cycle for HFDM04/05. TC\#1-\#3 are thermocouples placed within the oven during reaction. TC1 was placed in the oven air outside the retort. TC2 was placed in the retort air above the reaction fixture, and TC3 represents 6 thermocouples attached directly to the reaction fixture. Total thermal gradient across the reaction fixture was 3C at 210C, 5C at 400C and 7C at 650C. Gradient across the coil is expected to be about half these values.

\section{Insulation and Quench Heaters}

Coil radial ground insulation consisted of 3 layers of $0.125 \mathrm{~mm}$ ceramic sheet. A quench protection (strip) heater, consisting of 2 stainless strips bonded to a $0.100 \mathrm{~mm}$ thick piece of Kapton, was placed between the outer coil and the first sheet from the outer coil surface in each quadrant, with each strip placed approximately over the center of a current block.

\section{Coil Splicing and Impregnation}

A NbTi lead was soldered at the parting plane to each of the inner and outer leads using $70 \% \mathrm{~Pb}-30 \% \mathrm{Sn}$ solder. Coils were impregnated with CTD101K epoxy, in the same fixture that was used for reaction. The fixture was placed in a large oven and heated to $60 \mathrm{C}$ with the oven evacuated to 75 microns. The container of epoxy was heated to 55C and evacuated to about 115 microns. The epoxy flow rate into the coil was $0.04 \mathrm{~cm}^{3} / \mathrm{s}$. Total time for the impregnation process was approximately 7 hours. After impregnation, the fixture was placed into an oven and cured at 125C for 21 hours.

\section{Coil Size Measurements}

The thickness, width and flatness of the coils were measured after impregnation in the free state, as shown in Figure 4. Data from these three measurements are shown in Figure 5.
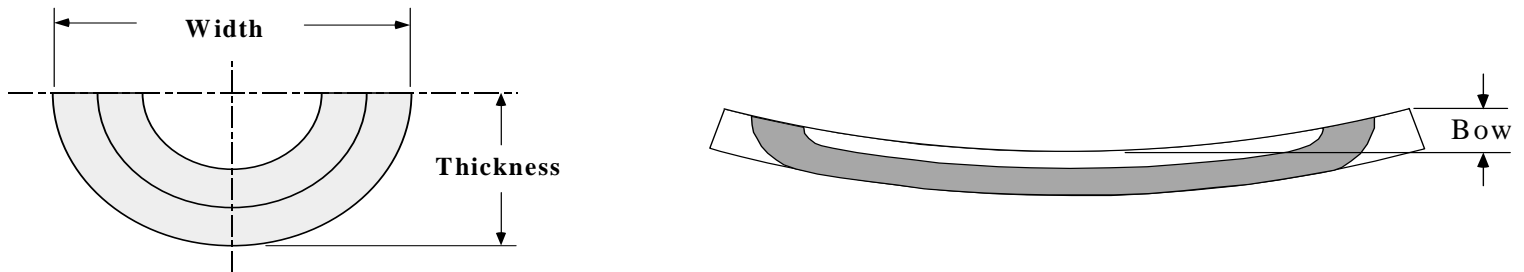

FIGURE 4. Thickness, width and flatness (bow) measurements 

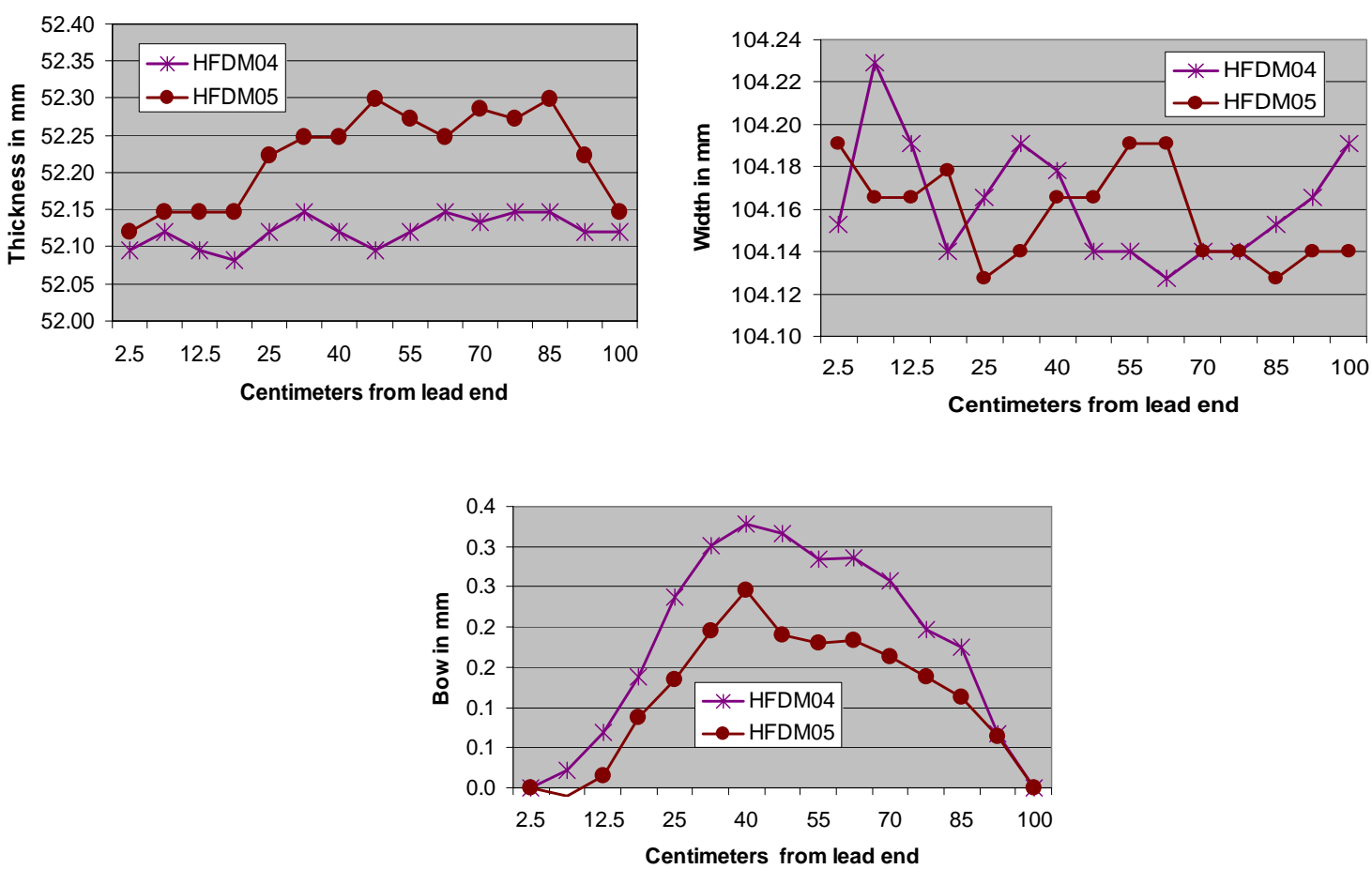

FIGURE 5. Thickness, width and flatness measurement data. Coil thickness is consistent in the coil body for each coil within $0.05 \mathrm{~mm}$, although HFDM05 coil is larger than HFDM04 one by $0.2 \mathrm{~mm}$, probably due to differences in cable thickness. Coil width is consistent both within each coil and between the coils within $0.1 \mathrm{~mm}$. Both coils are flat within $0.4 \mathrm{~mm}$ in free state. This is consistent with all Nb3Sn coils previously built at FNAL.

\section{Instrumentation}

Both capacitance and resistive beam strain gauges were used to measure coil azimuthal preload. General position of gauges with respect to the cross section is shown in Figure 6. Grey rectangles show the approximate position of the gauges, and black arrows show the direction in which force is being measured. Two longitudinal positions, in the body and at the lead end splices, were measured. A capacitance gauge was placed at each outer pole in the body. Resistive strain gauges were also bonded to the inside surface of the impregnated coil. These gauges were bonded to the current block nearest the pole on the inner layer of HFDM04, and to the middle current block of the inner layer on HFDM05.

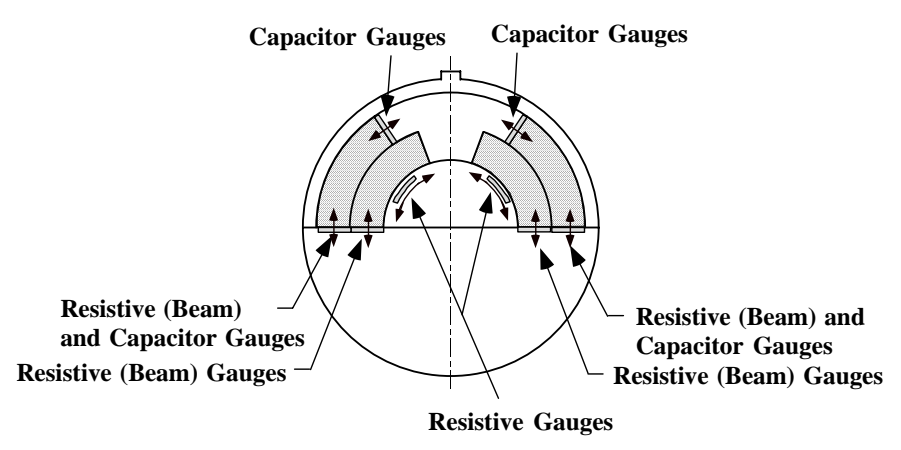

FIGURE 6. Gauge Positions.

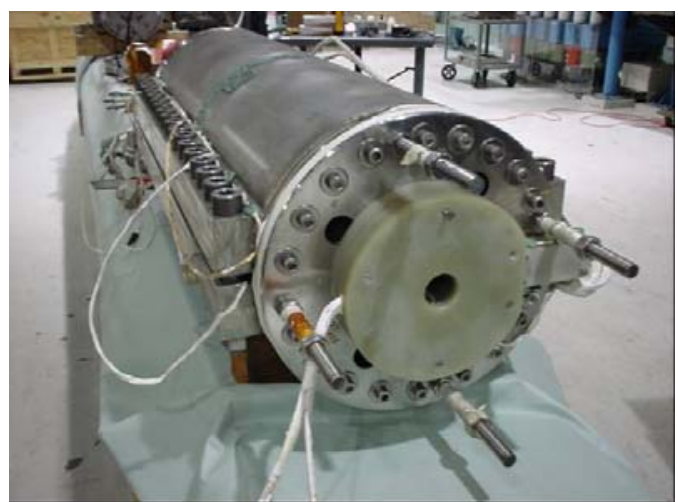

FIGURE 7. Completed Mirror (Lead end shown). 
Resistive gauges were also mounted onto the surface of the skin near the longitudinal center, positioned to measure skin azimuthal strain. They were mounted on both the upper and lower skins, at azimuthal positions of 30, 60 and 90 degrees from the yoke/skin gap.

\section{Assembly and Coil Pre-load}

The iron mirror blocks were installed into the lower yoke. The coil was then placed onto the mirror and the upper yoke blocks installed. After pressing vertically until the nominal yoke gap was achieved, the press position was locked and the yoke clamps inserted. Press pressure was then released. Skin halves were placed around the yoked assembly and bolted together. Bolting was done in several steps, while stress in the coil and end spacers was monitored by the skin gauges. These gauges showed that stress in the skin after bolting was approximately $90 \mathrm{MPa}$. After the skins were installed, $50 \mathrm{~mm}$ thick end plates were bolted to the skin ends. Axial preload may be applied through end preload bolts attached to the end plates. Initially, end loading was not applied to either mirror. After the first test, HFDM05 was retested with a total end load of $7000 \mathrm{~N}$ applied.

Pressure to the coil during assembly is applied by the press to the yoke halves, reducing the yoke gap and applying radial stress to the coils through the spacers. The spacers are loaded radially, but not azimuthally, because there is a large azimuthal gap between the spacers and the mirror. Azimuthal compressive (hoop) stress is therefore applied to the coils, as the coil midplane is pressed against the stainless steel strips. The yoke clamps are inserted and the press pressure is released, transferring the press pressure to tensile stress in the yoke clamps. The inside surfaces of the yoke clamps are now in contact with the yoke, and there is still a gap between the yoke halves. The skin is bolted onto the yoke, applying preload to the coils, relieving some but not all of the stress from the yoke clamps. The skin is now in azimuthal tension, and the inside surfaces of the yoke clamps are still in contact with the yoke. Stress on the spacers and coil is unchanged.

During cooldown, all components shrink (at different rates). The yoke gap remains open, and the tensile stress in the skin and yoke clamps increase.

During excitation, forces are applied radially outward by the coils at the parting plane. These forces are contained by the skin and the yoke clamps.

Readings of strain gauges after the magnet was assembled are shown in Table 2. Mirror magnet, completed with skin and end plates, is shown in Figure 7.

The details of HFDM04 and HFDM05 fabrication are reported in ${ }^{6,7}$.

TABLE 2. Strain Gauge Readings.

\begin{tabular}{|l|c|l|l|l|}
\hline Gauge Type & No. of Gauges & Units & HFDM04 & HFDM05 \\
\hline Resistive Beam Straight section midplane Inner coil & 2 & $\mathrm{MPa}$ & $56^{*}$ & $58^{*}$ \\
\hline Resistive Beam Straight section midplane Outer coil & 2 & $\mathrm{MPa}$ & $51^{*}$ & failed \&47 \\
\hline Resistive Beam Splice area midplane Inner coil & 1 & $\mathrm{MPa}$ & failed & 28 \\
\hline Resistive Beam Splice area midplane Outer coil & 1 & $\mathrm{MPa}$ & 87 & 22 \\
\hline Capacitor Gauge Straight Section midplane Outer coil & 2 & $\mathrm{MPa}$ & failed \& 73 & failed \& 60 \\
\hline Capacitor Gauge Pole Straight Section Outer coil & 2 & $\mathrm{MPa}$ & 25 and 60 & both failed \\
\hline Resistive Gauge on bore in Straight Section & 2 & $\mathrm{MPa}$ & $74^{*}$ & $45^{*}$ \\
\hline Resistive Gauge on Bore near Non-lead End & 2 & $\mathrm{MPa}$ & $73^{*}$ & $20^{*}$ \\
\hline
\end{tabular}

*Average of 2 gauges with similar readings is shown. 


\section{TEST RESULTS AND CONCLUSIONS}

Both mirror magnets were tested at 4.5 and $2.2 \mathrm{~K}$. Complete test data are reported in references $^{8.9}$. Magnet quench history for HFDM04 and HFDM05 is plotted in Figure 8.

Both magnets exhibited practically no training at $4.5 \mathrm{~K}$. Quench current, as shown in Figure 8, did not exceed $7 \mathrm{kA}$ in HFDM04 and $8 \mathrm{kA}$ in HFDM05. After training at $4.5 \mathrm{~K}$ the magnets were trained also at $2.2 \mathrm{~K}$. In HFDM04 instead of increasing the quench currents decreased to $\sim 5 \mathrm{kA}$. Quench behavior of HFDM05 at $2.2 \mathrm{~K}$ was quite the same as it was at 4.5 $\mathrm{K}$ with slightly increased quench current plateau. After increasing the temperature back to 4.5 $\mathrm{K}$ the quench currents in both magnets went back to the same current range observed prior of quenching it at $2.2 \mathrm{~K}$. In both magnets the resistive gauges mounted on the inner-layer coil surface and the resistive beam gauges installed in the mid-plane in the coil body showed elastic strain dependence as a function of the Lorentz force.

After performing a thermal cycle and adjusting the magnet end support, HFDM05 was re-tested. However, the quench current values remained at the same level.

In HFDM04 all the training quenches but one at $4.5 \mathrm{~K}$ were located in the inner-layer mid-plane block. The $2.2 \mathrm{~K}$ quenches were in the inner-layer middle block region. In HFDM05 the training quenches at $4.5 \mathrm{~K}$ and $20 \mathrm{~A} / \mathrm{s}$ ramp rates were located in the outer coil. Higher ramp rate quenches started both in the inner and outer coils almost simultaneously. The locations of training quenches at $2.2 \mathrm{~K}$ were alternated between the inner and outer coils. During the test many voltage spikes were detected in both magnets ${ }^{10}$.

The measured RRR value for HFDM04 was 18 for the inner coil and 20 for the outer coil. The measured RRR value for HFDM05 was 55 for the inner coil and 30 for the outer coil.

Low quench current values, erratic quench behavior and intensive voltage spikes indicate that the magnets were likely to be limited by conductor instabilities.
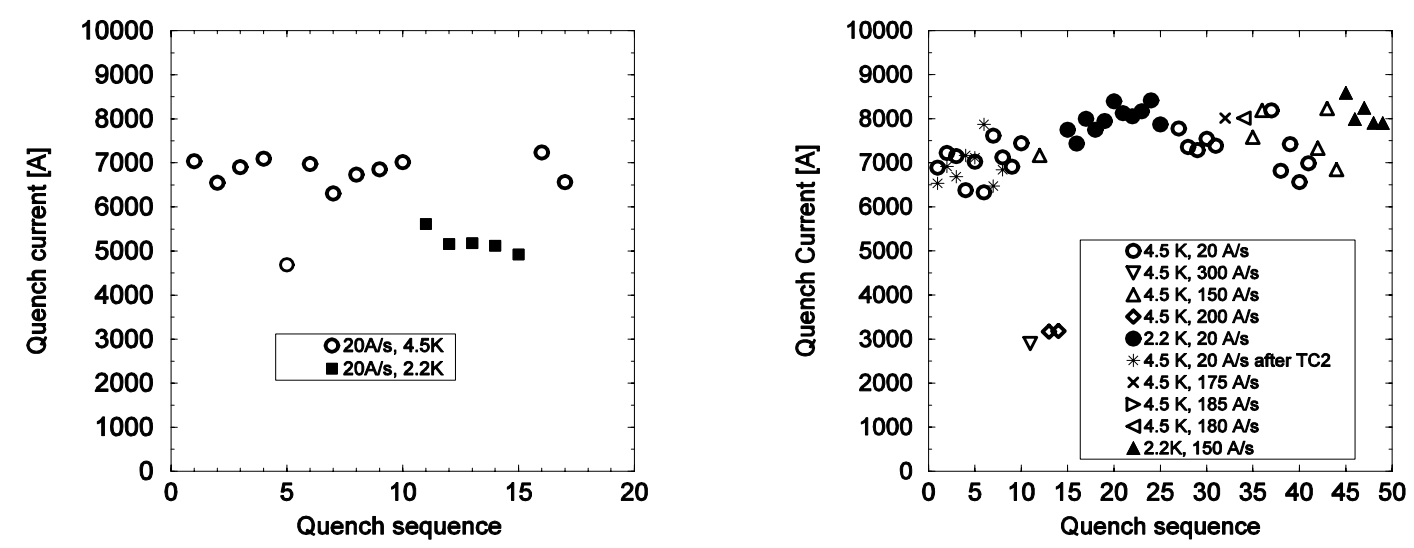

FIGURE 8. Quench performance for HFDM04 (left) and HFDM05 (right).

Round and extracted from cable strand samples were placed into the reaction retort during coil reaction. Results of witness sample tests at $4.2 \mathrm{~K}$ are summarized in Tables 3 and 4 . Sample RRR data are consistent with magnet RRR values. In spite of relatively high RRR most of the samples showed premature quenches at $12 \mathrm{~T}$ and even at $15 \mathrm{~T}$ indicating on strand stability problem. At least one witness sample for each magnet demonstrated premature quenches at very low current level. Analysis of cable cross-sections confirmed the presence of strands in each cable with merged deformed sub-elements due to broken diffusion barrier. 
TABLE 3. Witness sample tests from the coil used in HFDM04.

\begin{tabular}{|c|c|c|c|c|c|}
\hline Sample \# & Sample State & Barrel Material & Ic (12T), A & Ic (15T), A & RRR \\
\hline 1 & Extracted & Stainless Steel & 422 & 211 & 7 \\
\hline 2 & Extracted & Titanium & $382^{*}$ & $228^{*}$ & 16 \\
\hline 3 & Extracted & Stainless Steel & $385^{*}$ & 210 & 14 \\
\hline 4 & Extracted & Titanium & $176^{*}$ & $137^{*}$ & 8 \\
\hline 5 & Extracted & Titanium & $336^{*}$ & 232 & 33 \\
\hline 6 & Extracted & Titanium & $106^{*}$ & $76^{*}$ & \\
\hline
\end{tabular}

TABLE 4. Witness sample tests from the coil used in HFDM05.

\begin{tabular}{|c|c|c|c|c|c|}
\hline Sample \# & Sample State & Barrel Material & Ic (12T), A & Ic (15T), A & RRR \\
\hline 1 & Extracted & Titanium & 325 & 167 & 64 \\
\hline 2 & Round & Stainless Steel & 360 & 177 & 274 \\
\hline 3 & Extracted & Titanium & $352^{*}$ & 185 & 68 \\
\hline 4 & Extracted & Stainless Steel & $123^{*}$ & $96 *$ & 35 \\
\hline 5 & Round & Titanium & $297^{*}$ & $219 *$ & 64 \\
\hline 6 & Round & Titanium & $362^{*}$ & $209 *$ & 36 \\
\hline 7 & Round & Titanium & $375^{*}$ & $203^{*}$ & 80 \\
\hline
\end{tabular}

* premature quench

Neither of the mirrors made with RRP cable performed at the level expected, although magnet structure and construction techniques were nearly identical to previous successful PIT mirrors and dipoles ${ }^{4}$. Instabilities in the conductor are considered as a possible cause. The work on improvement of RRP strand stability will continue in collaboration with OST.

\section{ACKNOWLEDGEMENTS}

This work is supported by the Department of Energy. The authors thank M. Whitson, J. Alvarez , L. Mokhov and A. Bianchi for technical expertise during magnet fabrication.

\section{REFERENCES}

1. G. Ambrosio et al., "Development of the $11 \mathrm{~T} \mathrm{Nb}_{3}$ Sn Dipole Model at Fermilab", MT-16, Tallahassee, FL, September 1999. IEEE Transactions on Applied Superconductivity, v. 10, No. 1, March 2000, p.298.

2. S. Feher et al., "Test Results of Shell-type Nb3Sn Dipole Coils”, IEEE Transactions on Applied Superconductivity, Volume 14, Issue 2, June 2004 Page(s):349 - 352

3. A.V. Zlobin et al., "R\&D of $\mathrm{Nb}_{3} \mathrm{Sn}$ Accelerator Magnets at Fermilab”, IEEE Transactions on Applied Superconductivity, Volume 15, Issue 2, June 2005 Page(s): 1113 - 1118.

4. A.V. Zlobin et al., "Development and Test of Nb3Sn Cos-theta Dipoles Based on PIT Strands", IEEE Transactions on Applied Superconductivity, Volume 15, Issue 2, June 2005 Page(s):1160 - 1163

5. D.R. Chichili et al., "Design, Fabrication and Testing of Nb3Sn Shell Type Coils in Mirror Magnet Configuration”, presented at the CEC/ICMC 2003, Alaska, September 22-25 2003.

6. R. Bossert. et al, "HFDM04 Production Report” Fermilab Technical Division Technical Memo TD-05-023.

7. R. Bossert et al, "HFDM05 Production Report" Fermilab Technical Division Technical Memo TD-05-038.

8. S. Feher et al, "HFDM04 Test Summary" Fermilab Technical Division Technical Memo TD-05-030.

9. S. Feher et al, "HFDM05 Test Summary", Fermilab Technical Division Technical Memo TD-05-031.

10. S. Feher et al., "Development and Test of Nb3Sn Cos-theta Magnets Based on RRP Strands," to be presented at the International Magnet Technology Conference, MT-19, Genova, Italy, 2005. 